\title{
Editorial: To the memory of John P. Klein
}

\author{
Niels Keiding • Per Kragh Andersen • \\ Mei-Jie Zhang
}

Received: 27 December 2014 / Accepted: 6 January 2015 / Published online: 7 February 2015

(C) Springer Science+Business Media New York 2015

This issue of Lifetime Data Analysis (LIDA) is dedicated to John P. Klein, a long-time member of LIDA's Editorial Board since 1994. John was born on December 30, 1950 and passed away on July 20, 2013 at the age of 62.

John spent his career as a hard-working biostatistician, honoring solid old-fashioned virtues in his working discipline, complete honesty and long-lasting friendships. After his Ph.D. in Statistics from the University of Missouri, Columbia in 1980 he spent 13 years at the Department of Statistics of the Ohio State University in Columbus, reaching the post of Professor of Statistics and Preventive Medicine and Statistical Director of The Ohio State University Comprehensive Cancer Center. In 1993 he returned to his native Milwaukee as Professor and Director of the Division of Biostatistics at the Medical College of Wisconsin and Statistical Director of the Center for International Blood \& Marrow Transplant Research (CIBMTR). John left a large publication list both in biostatistical methodology (primarily survival analysis) and cancer research, with focus on bone marrow transplantation for the last more than 20 years. He wrote an acclaimed textbook with Mel Moeschberger: Survival Analysis: Techniques for Censored and Truncated Data (Springer, 1997, Second Edition 2005), edited (with P.K. Goel) Survival Analysis: State of the Art (Kluwer, 1992) and before his death just finished editing (as chief editor, with H.C. van Houwelingen, J.G. Ibrahim and T.H. Scheike) the comprehensive Handbook of Survival Analysis (Chapman \& Hall, 2014), although he never saw the completed volume. John was single all his life but kept close contact with his mother and sister, both in Milwaukee.

John had studied survival analysis in his Missouri dissertation (supervised by A.P. Basu) and contacted us in Copenhagen in 1986 with a view to spending his upcoming sabbatical year 1987-1988 there. This lead to a long and fruitful collaboration, aided

\footnotetext{
N. Keiding $(\varangle) \cdot$ P. K. Andersen · M.-J. Zhang University of Copenhagen, Copenhagen N, Denmark e-mail: n.keiding@biostat.ku.dk
} 
by a sequence of joint R-01 Grants from the U.S. National Cancer Institute with co-PIs P.K. Andersen, N. Keiding, T.H. Scheike and M.J. Zhang, on complex survival analysis problems around Bone Marrow Transplantation. This led to both methodological developments and to overview articles aimed at a clinical audience.

The scientific director of CIBMTR, Dr. Mary Horowitz, praised his leadership, under which "the CIBMTR advanced the statistical science of clinical outcomes research in blood and marrow transplantation. His expertise and insistence on statistical rigor in all studies were key to establishing the CIBMTR's reputation for high quality clinical research. In addition to his work with the CIBMTR, Dr. Klein collaborated with investigators in the Medical College of Wisconsin's Departments of Emergency Medicine and Orthopedics and with investigators at Marquette University's School of Engineering. Dr. Klein was a dedicated and respected graduate and medical school educator and mentor. He advised more than 30 graduate students and in 2005 was named Outstanding Graduate School Mentor by the Medical College of Wisconsin."

The idea of dedicating a separate issue of LIDA to the memory of John Klein came from Chief Editor Mei-Ling Ting Lee, and we were happy to accept this opportunity to help preserve the good memories of John's personality and his scientific achievements. We hoped to assemble a group of authors who could represent John's scientific areas as well as the stages of his career, and we were fortunate that everybody approached were happy to contribute.

From the mainstream of survival analysis come Lawless and Rad with a contribution on how to handle Markov multistate models, when transitions are not observed directly, only state occupations are observed at a discrete skeleton.

van Houwelingen and Putter (Leiden, The Netherlands) tackle a very common issue in applied survival analysis: how to express the result of the analysis in terms of a statement of survival prediction. These authors have made important contributions to this practical problem by emphasizing that any prediction of interest is a statement about events some positive time away from now. One natural procedure then becomes to use an ordinary Cox regression, but omit any information after the prediction origin $t_{0}$ by artificially censoring all follow-up at that point. In the present contribution they compare this approach (called 'stopped Cox' regression) with two other approaches: pseudo-values and direct binomial regression.

In competing risks methodology much recent effort has been spent on developing direct models for the cumulative incidence models, the most widely used being the proposal due to Fine and Gray (1999). Li and Zhang from the Medical College of Wisconsin (MCW), with Scheike from Copenhagen, have developed focused tests of goodness-of-fit tests for this model, based on cumulative sums of residuals and targeting the most important types of deviation from fit.

Many collaborative clinical trials nowadays have regular (annual, biannual) group meetings to monitor the progress of the trial and decide on action if there are remarkable developments regarding efficacy or safety. The so-called group sequential designs are tailored to this practical situation, so that the statisticians can deliver advice to the committees without violating the family-wise error rate. Logan (MCW), with Mo, have developed a variant of this problem for testing the null hypothesis that survival curves are identical after some specified time point. 
The most serious type of censoring is current status data, where all life times are censored, for each life time we only know whether it is smaller or larger than some given value. Sun (from the University of Missouri where John Klein did his Ph.D.), with Zhao, Hu, Ma and Wang, have studied regression analysis of current status data in the situation where censoring may be informative.

The ubiquitous Cox regression model invites the use of hazard ratios as effect measures, and these have now become rather dominant in many applications of survival analysis, so much so that Hernan (2010) felt it necessary to recommend restraint of this practice in observational epidemiology, on the premise that heterogeneity may often generate violation of the proportional hazards assumption necessary for many application of hazard ratios. Chen, Zeng, Ibrahim, Chen, Pan and Xue take a different approach, to derive a transformation family of weighted hazard ratio measures generalizing an earlier proposal by Schemper et al. (2009), continuing and elaborating the motivation for such efforts from that paper.

Despite their obvious relevance for human genetic epidemiology of twins, bivariate survival models are still in need of refinement and sophistication for this application. Scheike, Holst and Hjelmborg have developed tools allowing the distinction of dependence between the lifetimes within a twin pair early versus late in life.

Per Kragh Andersen (Copenhagen) and Anders Skrondal (Oslo) contribute to the long discussion regarding further formalization of Rothman's so-called sufficient cause model for 'biologic' interaction by developing a competing risks framework.

Finally, Wolfe, with Gemayel and Stasny (all at Columbus, OH, where John Klein spent the important early part of his career at Ohio State University) contribute a Bayesian treatment of ranked set sampling.

We are grateful to Chief Editor Mei-Ling Ting Lee for her initiative and help throughout and to Divyalochany Thangavel of the Springer staff for much practical help with the editorial systems.

\section{References}

Fine JP, Gray RJ (1999) A proportional hazards model for the subdistribution of a competing risk. J Am Stat Assoc 94:496-509

Hernan MA (2010) The hazards of hazard ratios. Epidemiology 21:13-15

Schemper M, Wakounig S, Heinze G (2009) The estimation of average hazard ratios by weighted Cox regression. Stat Med 28:2473-2489 